\title{
Time-variability of the Crab Nebula wisps
}

\author{
Anatoly Spitkovsky and Jonathan Arons \\ Astronomy Dept., University of California, Berkeley, CA 94720, USA
}

\begin{abstract}
We present results of time-dependent numerical modeling of the internal structure of the collisionless shock terminating the pulsar wind in Crab Nebula. We treat the equatorial relativistic wind as composed of ions and electron-positron plasma with an embedded toroidal magnetic field. Relativistic cyclotron instability of the ion ring downstream from the shock is found to launch outward propagating magnetosonic waves. Due to the fresh supply of ions crossing the shock, the time-dependent process achieves a limit-cycle pattern, in which the waves are launched with periodicity on the order of the ion Larmor time. Compressions in magnetic field and pair density associated with these waves as well as their propagation speed qualitatively reproduce the features observed in the wisps.
\end{abstract}

The interpretation of wisps in Crab Nebula as markers of internal structure of collisionless shock in equatorial pulsar outflow has been successful in explaining average separation of the wisps and their relative brightness (Gallant $\&$ Arons, 1994). In this model, the first and second wisps are understood as compressions in $e^{ \pm}$pair plasma due to turning points in the $E \times B$ drift orbit of the ions. However, such coherent orbits are unstable to bunching and emission of magnetosonic waves which in turn tend to scramble up the orbits. This realization together with the availability of time sequence of HST images of the wisp region (Hester, 1996) had prompted us to modify the model of Gallant \& Arons to include the time-dependence of the flow.

Because of the small electron to proton mass ratio, the scale of ion dynamics is much larger than that of pairs. This means that we can numerically model the pair component of the wind as a relativistic 1D MHD fluid while simulating ions as particles using 2D PIC. Coupling between the two components is mediated by the Lorentz force and charge- and current-neutrality conditions. The boundary conditions are MHD shock on the left and a non-reflective outflow on the right. The latter condition allows to concentrate on the interesting region around the pair shock instead of modeling the whole nebula.

The main result of the time-dependent simulations is the development of a limit-cycle behavior in the first post-shock ion loop. This cycle consists of phase bunching and emission of magnetosonic waves, and repeats itself with a period of $\sim 0.5 \mathrm{~T}_{\mathrm{Li}}$ for a flow with adiabatic index $\Gamma=4 / 3$. Here $\mathrm{T}_{\mathrm{Li}}$ is the ion Larmor period in the post-shock wind. The increase in magnetic field due to traveling magnetosonic perturbations triggers bunching in the stream of fresh ions that crosses the shock, thus initiating the next cycle (figure 1). The resulting magnetic field profile (and hence synchrotron emission brightness) 
retains overall double peaked shape determined by turning points of the orbit, and has a magnetosonic wave that emanates from the first peak and propagates past the second peak with a period of emission $\sim 0.5 \mathrm{~T}_{\mathrm{Li}}$.
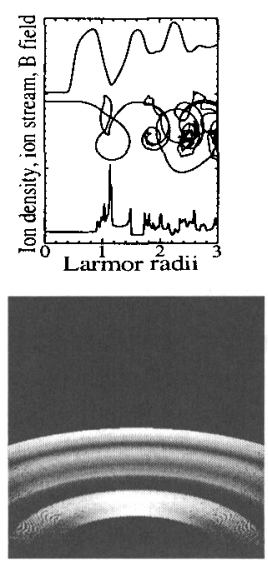
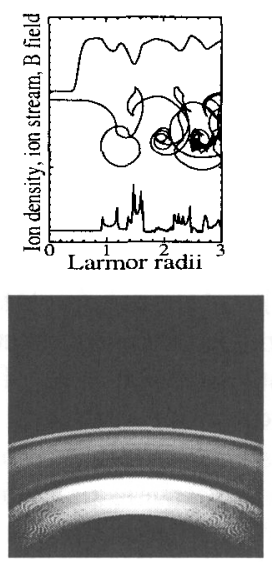
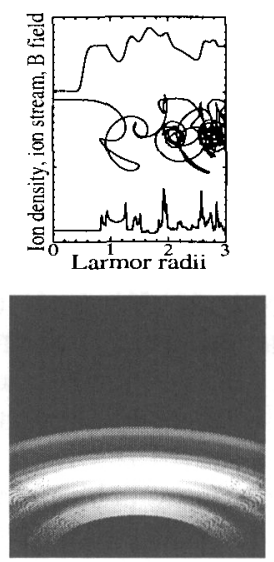
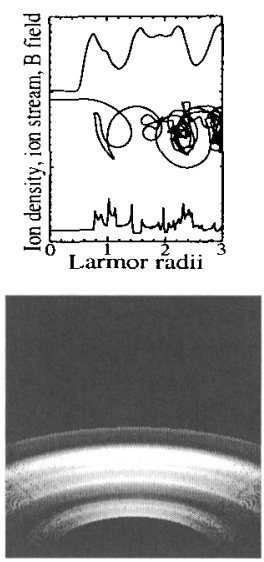

Figure 1. Top row: Pair fluid is "stirred" by the instability of ion loop. New waves are emitted with every turn of the bunched "knot" on the first loop. Bottom row: Simulated surface brightness.

By fitting parameters of ion loop orbit to match the observed mean separation of the wisps we infer the upstream ion Larmor radius to be $0.1-0.2 \mathrm{pc}$. The timescale of variability in the wisps is then predicted to be $4-8$ months. The first wisp will appear to brighten and then emit a wave towards the second wisp once every 4-8 months. This wave propagates with magnetosonic speed on top of the flow velocity, or $\sim 0.62 c$. When projected on the plane of the sky the wave speed is in the range $0.5 c$ to $0.66 c$ for inclination angles of pulsar equatorial plane between $60^{\circ}$ and $70^{\circ}$. As the wave approaches the location of wisp 2 , this region will brighten up. Beyond the first ion loop, the wave will scatter on the chaotic background of ions that underwent full cyclotron instability. That will make wisp 2 appear as having short wavelength filamentary structure.

This description is consistent with a series of HST snapshots of the nebula. While overall wisps 1 and 2 remain well separated and identifiable, both are very dynamic. A wisp-like feature is seen propagating from wisp 1 to wisp 2 with the speed of $\sim 0.55 c$. Observations separated by less than a few weeks would provide enough information for identification of moving features, but current data does not cover a full period of variability. Combined with earlier snapshots the period of emission of waves from wisp 1 can be estimated to be 5-6 months. Our theory then seems to account well for both the averaged and the time-varying behavior of the wisps. A detailed description of this model and comparison with the data will be presented elsewhere (Spitkovsky \& Arons, in preparation).

\section{References}

Gallant, Y. A. \& Arons, J. 1994, ApJ, 435, 230

Hester, J. J. http://oposite.stsci.edu/pubinfo/pr/96/22.html 